\title{
ILIA CHAVCHAVADZE ABOUT EXTERNAL TRADE AND MODERNITY
}

\section{ZURABI MTCHEDLISVILI}

\section{PhD student}

Ivane Javakhishvili Tbilisi State University, Georgia

zuramchedlishvili728@gmail.com

Abstract. The works of Ilia Chavchavadze - a prominent Georgian public figure of the nineteenth century and one of the founders of the Georgian National Economic Doctrine - are still relevant. His name grows stronger and stronger over time. Even in modern Georgia, Ilia Chavchavadzess name is the most often used to prove the validity of oness own opinion.

The article discusses Ilia Chavchavadzess views on international trade and trade policy and draws parallels with modernity. He researched the essence, causes, directions, and consequences of international trade and trade policy, and in 1887 he devoted a collection of articles entitled «Customs policy in Europe, free-trading and protection». He explained why countries start trading, that international trade is very important and without it there can be no economic specialization - the international division of labor. In his research, Ilia Chavchavadze depthly discussed the directions of trade policy - liberal and protectionist. He discussed the arguments of the both directions proponents on the advantages of strengthening the country and the economy. Ilia Chavchavadze supported Liberal Trade Policy, because he believed that the main goal of any economic policy should be to maximize the well-being of society.

Ilia Chavchavadze has raised another very important issue in this research. He pointed out at the end of the nineteenth century that economic policy is not always based on economic rationalism, economic science, and that economic policy makers might have completely different motivations.

Ilia Chavchavadze believed that for the development of the country it was necessary to establish and deepen international - including trade and economic relations. According to llia, the start of the countryss international economic relations requires great care and attention, it is very important to choose reliable, trustworthy partners. In 2006, Georgia experienced the negative consequences of a relationship with an unreliable trading (and not just a trading) partner. Russia banned the import of Georgian wines on March 27, 2006, and also banned the import of Georgian mineral waters in May. The ban has had a very negative impact on Georgian exports, as well as on viticulture and winemaking.

Finally, it can be said that Ilia Chavchavadze was well versed in the essence and directions of trade policy; He was well aware that it was very important for the country to choose a logical, rational economic policy for its economic strengthening; He discussed in detail the characteristics of both directions, protectionist and freetherical, the pros and cons, the consequences of their possible impact on the economy and public welfare. With well-founded reasoning and argumentation, he supported liberal trade policy based on the principle of the well-being of society.

KEYWORDS: WELL-BEING OF SOCIETY; TRADE POLICY; LIBERALISM; PROTECTIONISM.

For citation: Mtchedlisvili, Z. (2020). Ilia Chavchavadze about external trade and modernity. Globalization and Business, №9, pp. 203-206 (In Georgian). https://doi.org/10.35945/gb.2020.09.025 


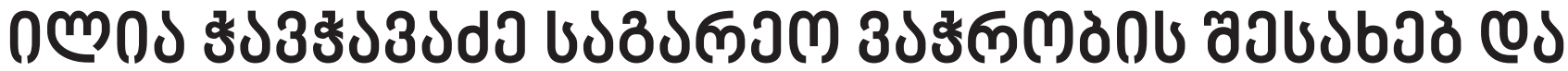

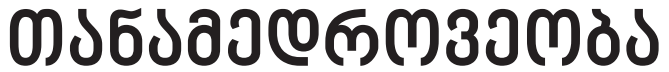

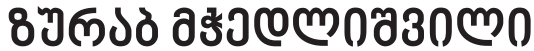

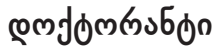

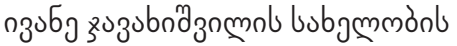

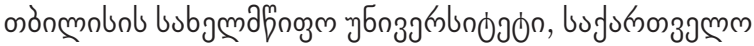

zuramchedlishvili728@gmail.com

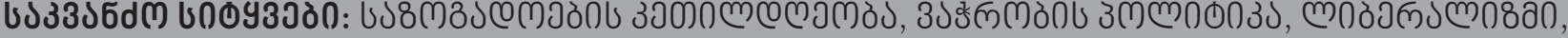

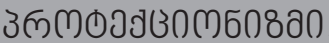

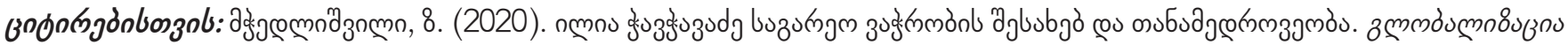
cou òn86glo, №9, 33. 197-206. https://doi.org/10.35945/gb.2020.09.025

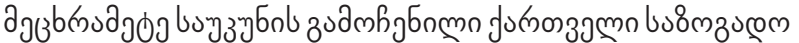

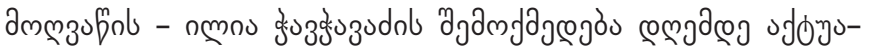

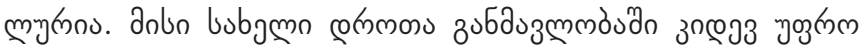

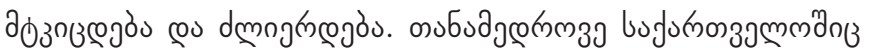

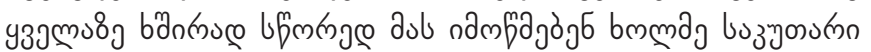

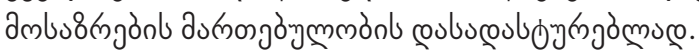

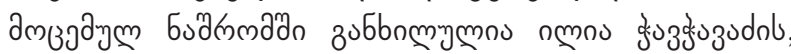

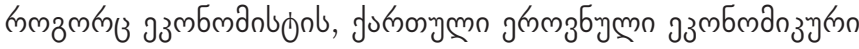

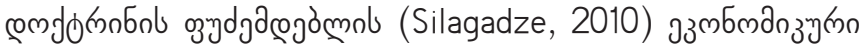

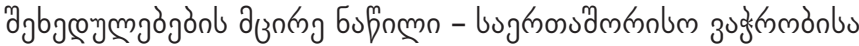

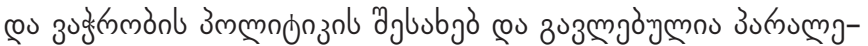

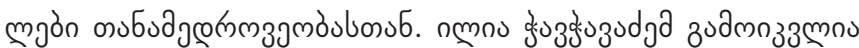

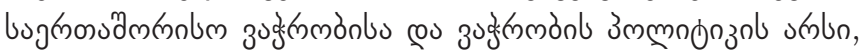

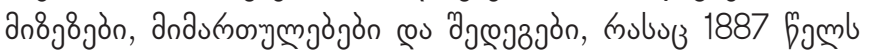

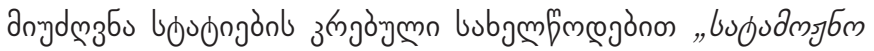

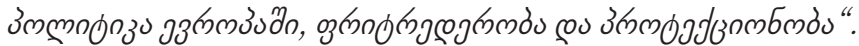

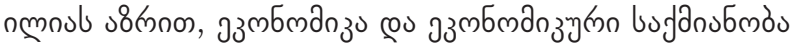

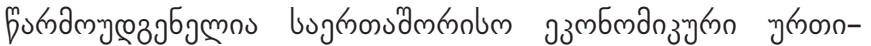

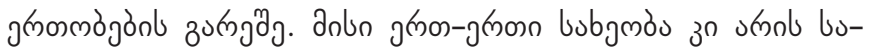

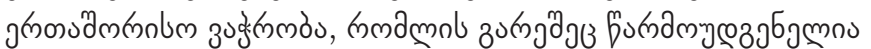

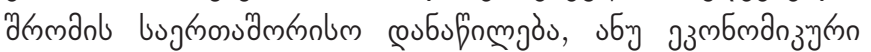

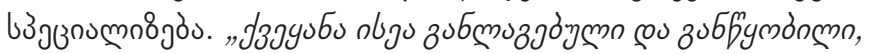

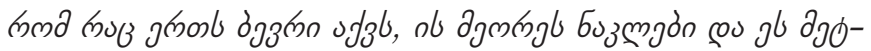

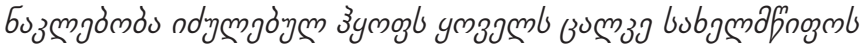

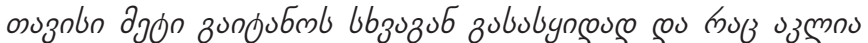

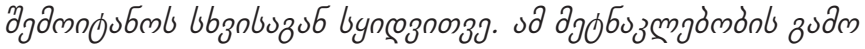

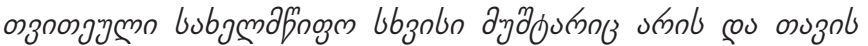

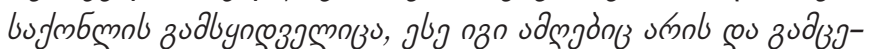

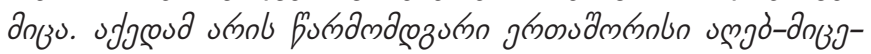

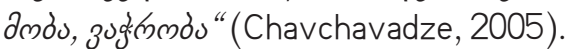

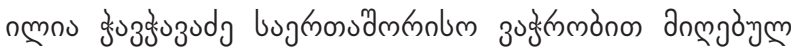

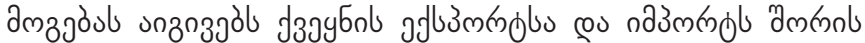

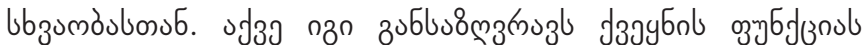

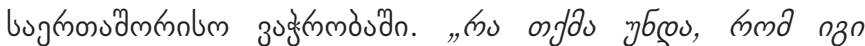

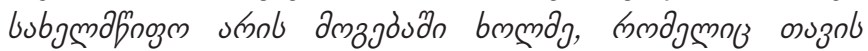

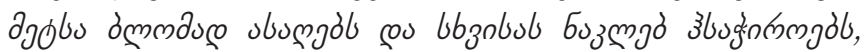

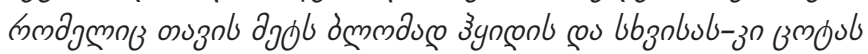

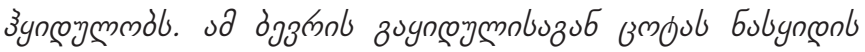

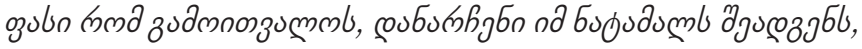

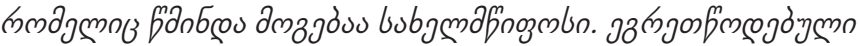

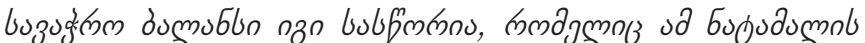

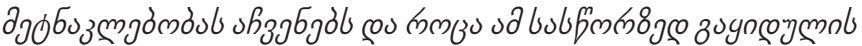

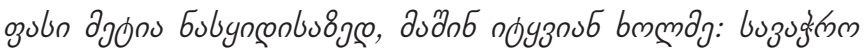

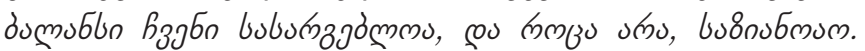

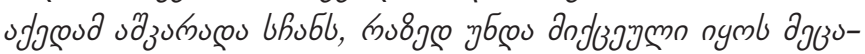

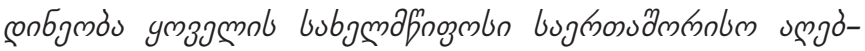

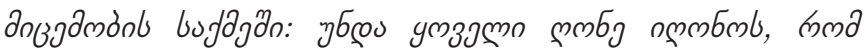

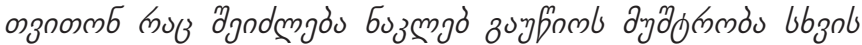

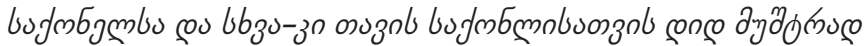
unonowls" (Chavchavadze, 2005).

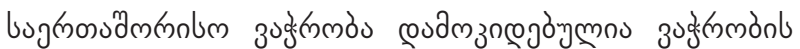

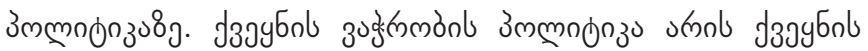

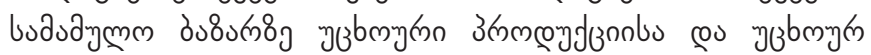

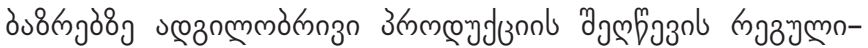

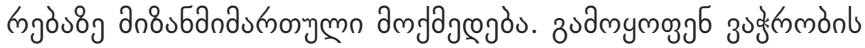

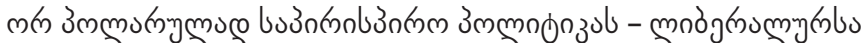

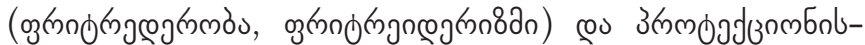

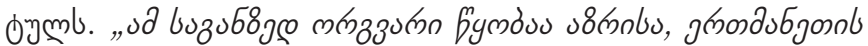

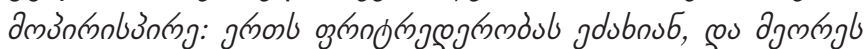

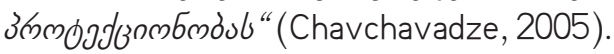

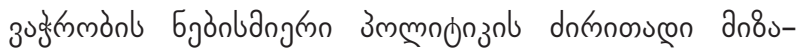

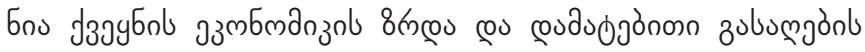




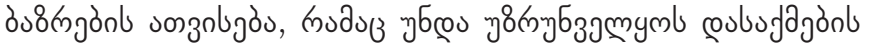

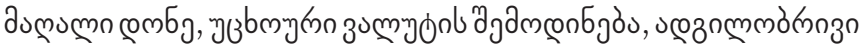

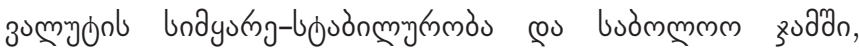

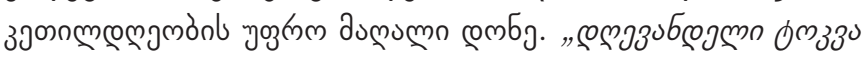

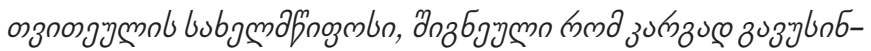

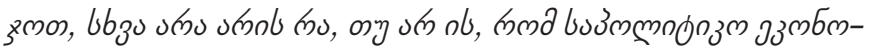

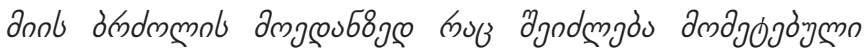

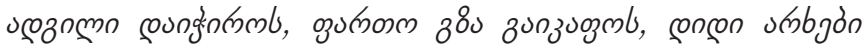

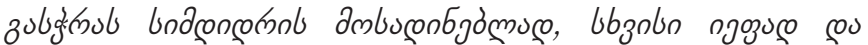

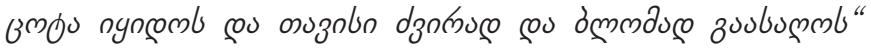
(Chavchavadze, 2005).

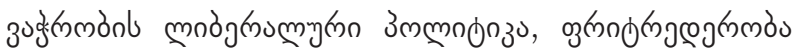

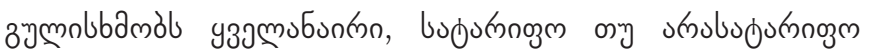

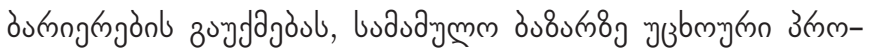

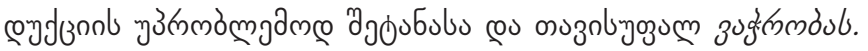

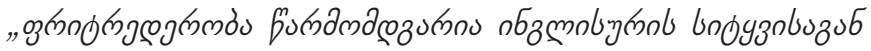

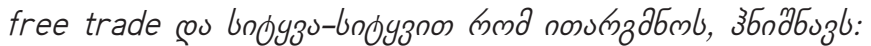

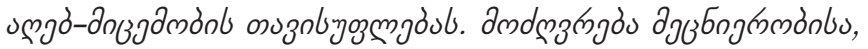

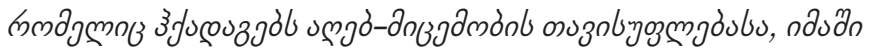

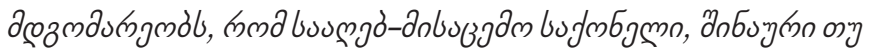

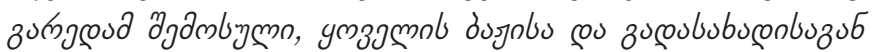

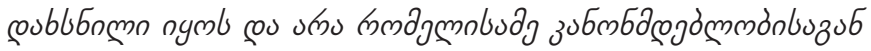

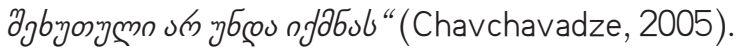

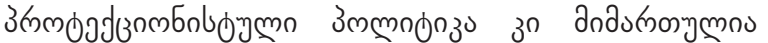

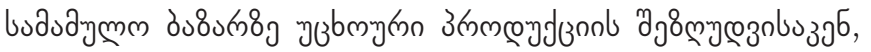

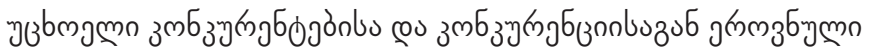

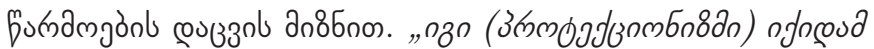

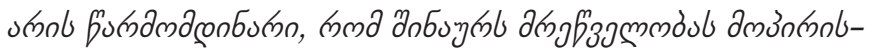

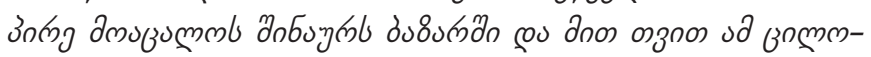

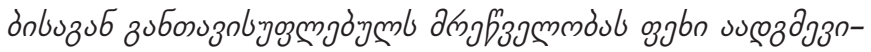

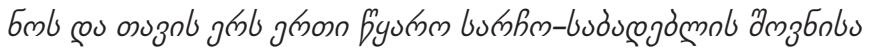
dmydugmb" (Chavchavadze, 2005).

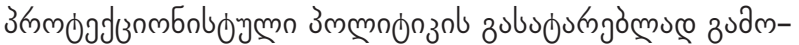

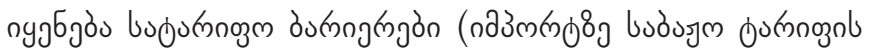

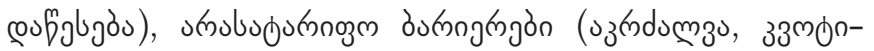

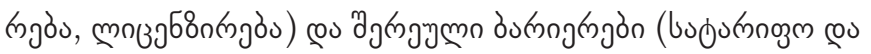

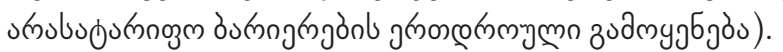

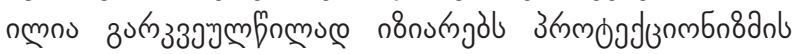

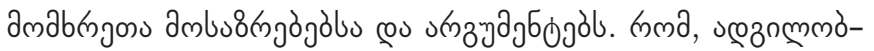

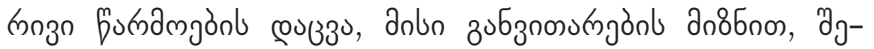

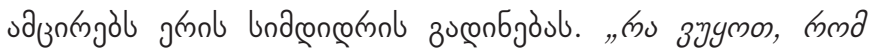

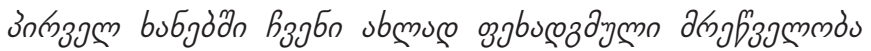

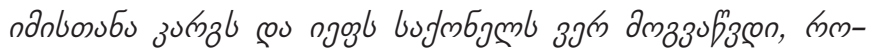

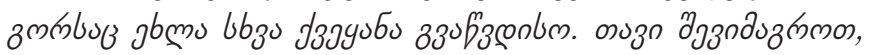

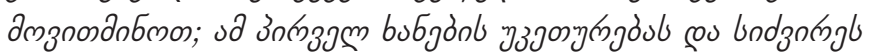

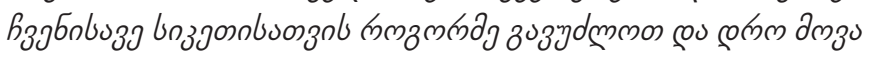
cou zn bu

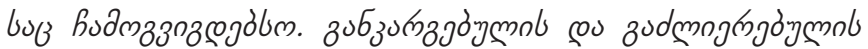

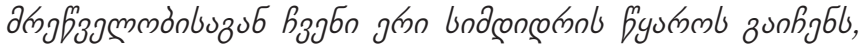

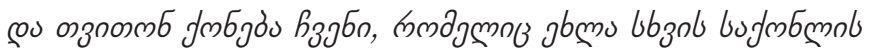

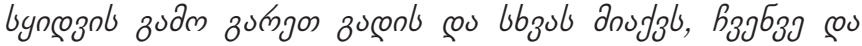

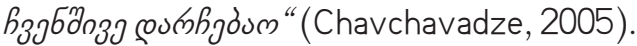

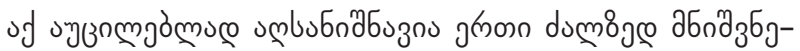

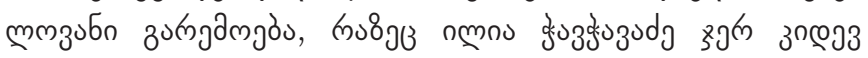

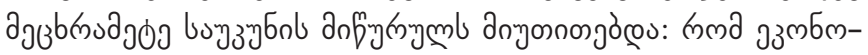

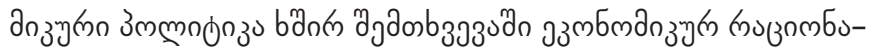

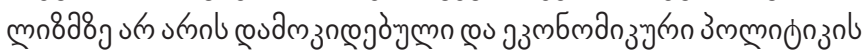

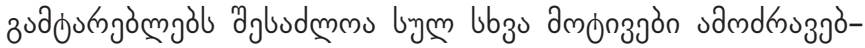

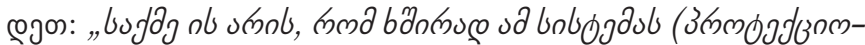

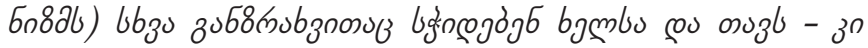

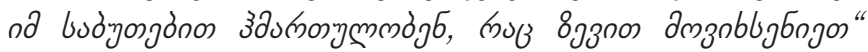
(Chavchavadze, 2005).

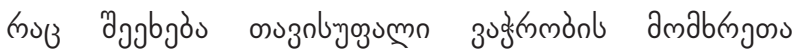

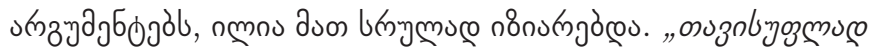

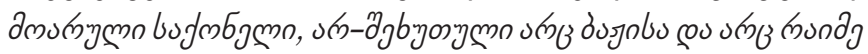

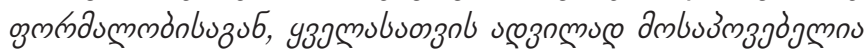

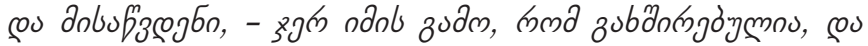

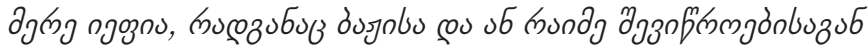

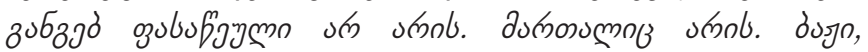

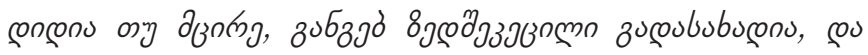

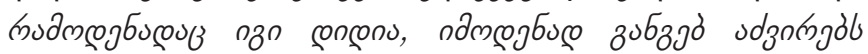

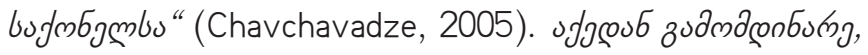

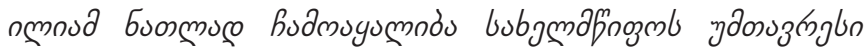

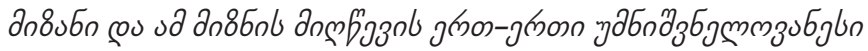
bu Iyu

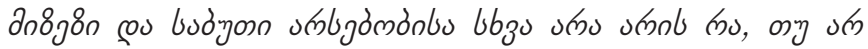

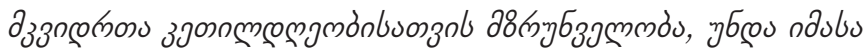

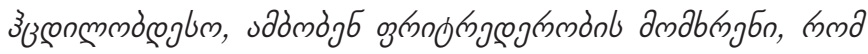

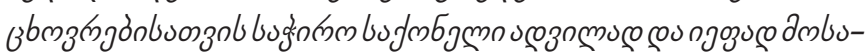

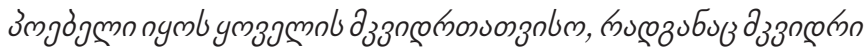

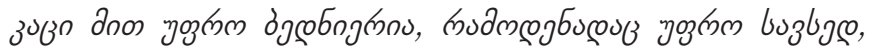

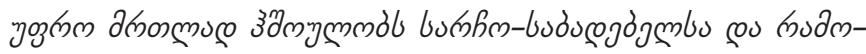

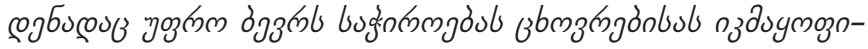

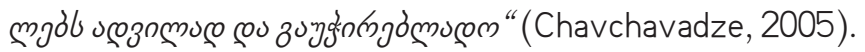

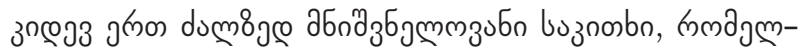

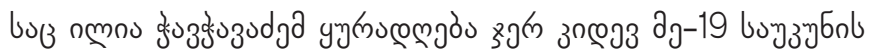

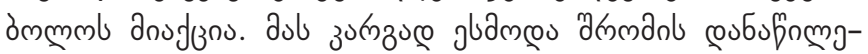

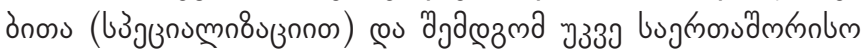

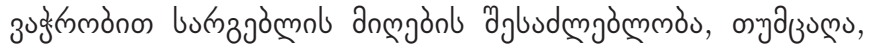

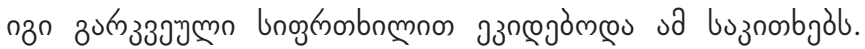

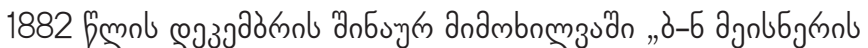

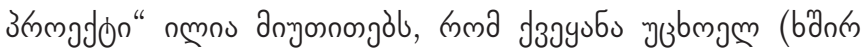

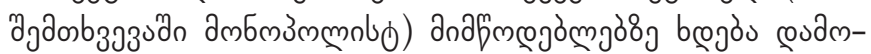

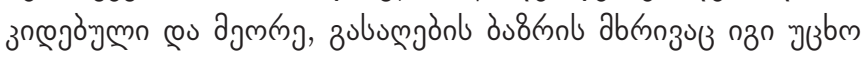

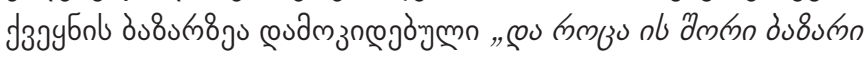

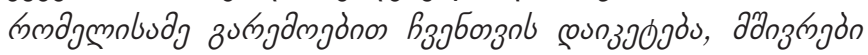

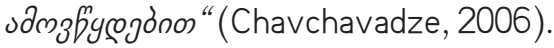

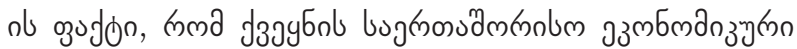

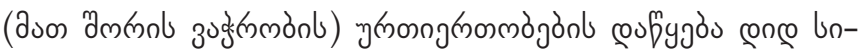

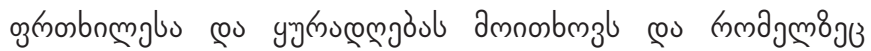




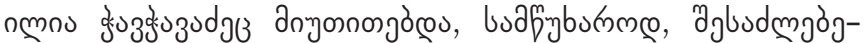

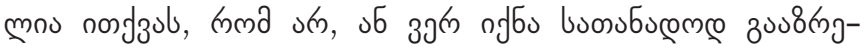

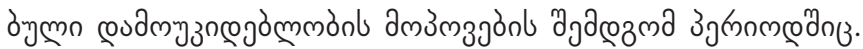

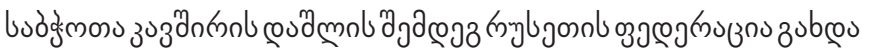

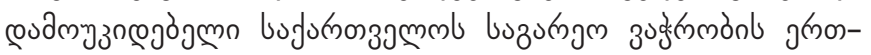

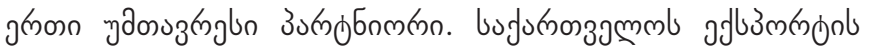

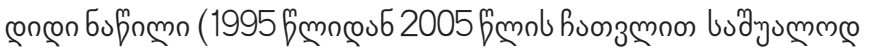

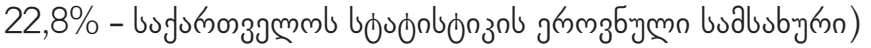

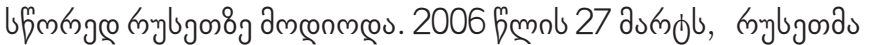

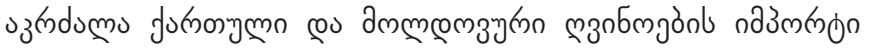

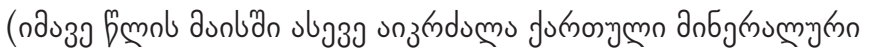

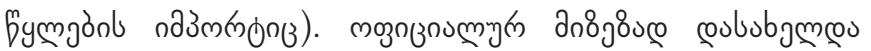

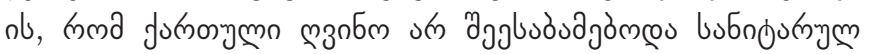

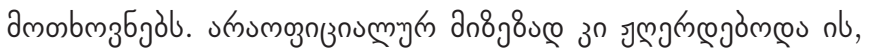

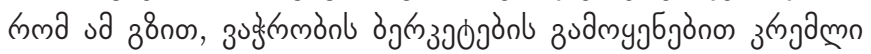

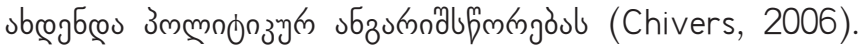

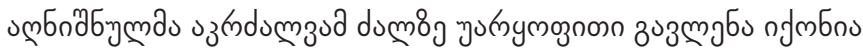

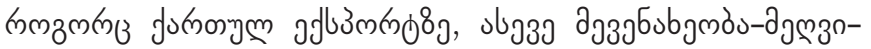

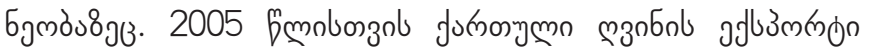

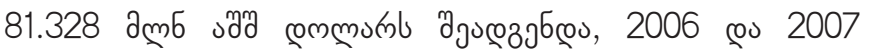

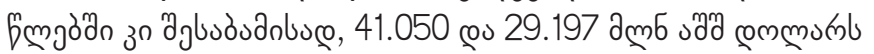

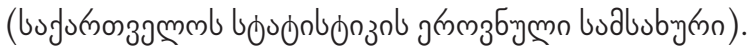

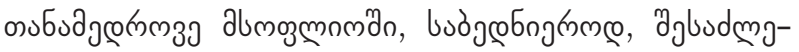

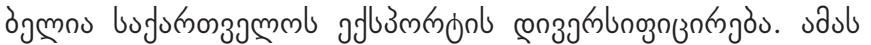

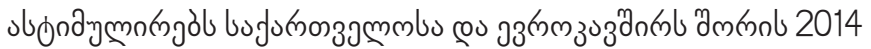

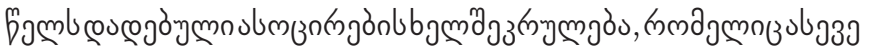

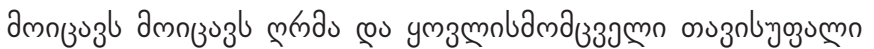

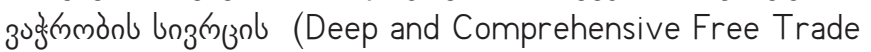

Area - DCFTA) hodmgumnodjòul (ASSOCIATION). umbnd-

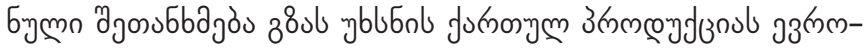
зงз

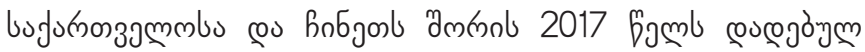

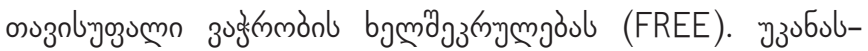

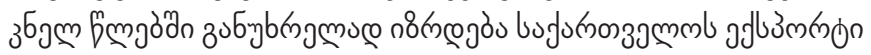

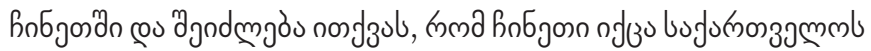

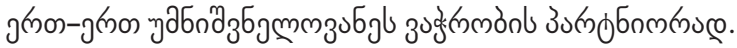

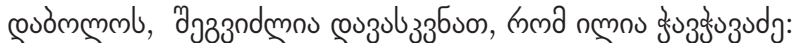

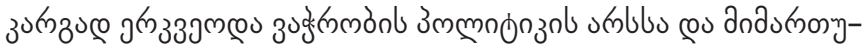

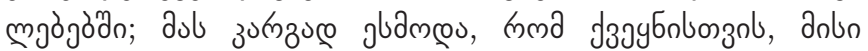

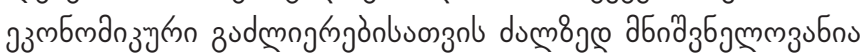

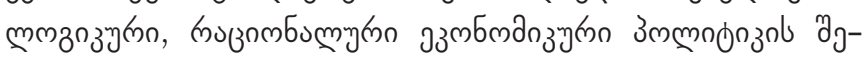

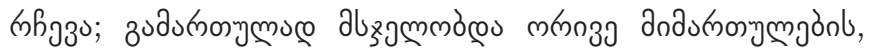

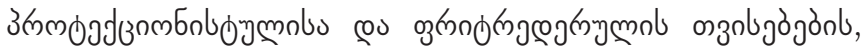

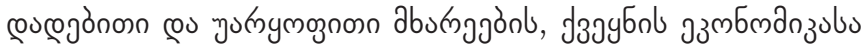

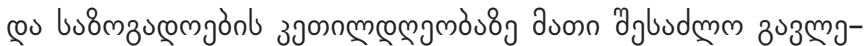

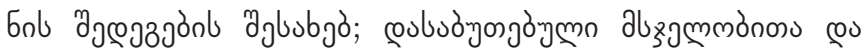

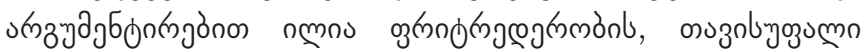

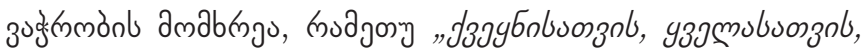

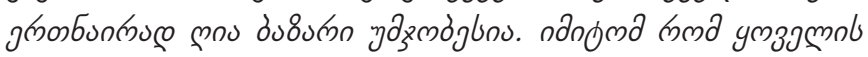

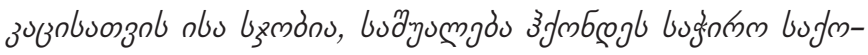

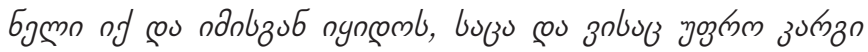

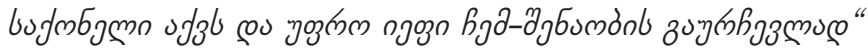

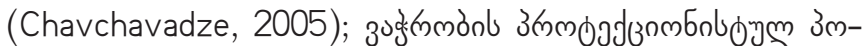

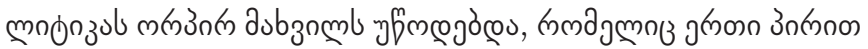

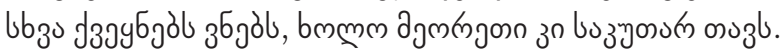

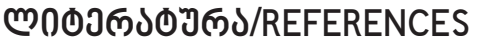

Chavchavadze, I. (2006). Customs policy in Europe, Free Trade and Protection. The Complete Collection of Twenty Volumes, Volume IX. Ilia Foundation, p. 385-401. (in Georgian).

Chavchavadze, I. (2005). Internal Review. Mr. Meisnerss project. Complete Collection of Twenty Volumes, Volume VII. Ilia Foundation, p. 26 - 79. (in Georgian).

Chivers, C. J. (2006). "A Russian ‘Wine Blockade) Against Georgia and Moldova”, The New York Times, APRIL 6. https://www. nytimes.com/2006/04/06/world/europe/a-russian-wine-blockade-against-georgia-and-moldova.html

Krugman, P., Obstfeld, M., Melitz, M. (2017) International Economics: Theory and Policy (10th Edition), Scienttific Editor and Editor-in chief of the Georgian Translation Silagadze A., Published by Tbilisi State University, Tbilisi (in Georgian).

Silagadze, A., Atanelishvili, T., Silagadze N. (2010) Economic Doctrines. Georgian National Academy of Sciences, Tbilisi (in Georgian).

Silagadze, A., Atanelishvili, T. (2010). Aspects of economic doctrines in Georgia. Peninsula University of Technology, Business and Informatics. San Francisco (USA).

Veshapidze, Sh. (2016) Ilia Chavchavadzess Economic Views on Open Economy Orientation: «Free trade and Protectionism», Tbilisi: Science and Life N1 (13), p. 47-52. (in Georgian).

ASSOCIATION AGREEMENT BETWEEN THE EUROPEAN UNION AND GEORGIA (in Georgian). https://matsne.gov.ge/ka/ document/view/2496959?publication $=0$

FREE TRADE AGREEMENT BETWEEN THE GOVERNMENT OF GEORGIA AND THE GOVERNMENT OF THE PEOPLE'S REPUBLICOF CHINA (in Georgian).

http://www.economy.ge/uploads/files/2017/foreign_trade/tavisufali_vachrobis_shetanxmeba_georgia_china/ shetankhmeba_2018/1_china_georgia_fta_geo.pdf 